\title{
Essential analytics in nursing education: Building capacity to improve clinical practice
}

\author{
Elizabeth Ritt \\ Benedictine University, Lisle, United States \\ Correspondence: Elizabeth Ritt. Address: Benedictine University, Lisle, United States. Email: eritt@ben.edu \\ Received: July 31, 2014 \\ Accepted: September 8, 2014 Online Published: September 14, 2014 \\ DOI : $10.5430 /$ jnep.v4n12p9 \\ URL: http://dx.doi.org/10.5430/jnep.v4n12p9
}

\section{Abstract}

There is an emerging use of analytics and big data to address major challenges and solve complex problems across the health care system. Analytics involves extensive and sophisticated use of data to describe, predict, prescribe, and compare data that drives decision making with the goal of improving results. The Patient Protection and Affordable Care Act coupled with collaboration among public and private organizations are driving the need for analytical data with the aim of improving the quality of health care and reducing costs to the consumer. The use of an analytical clinical decision framework is provided to assist members of the interprofessional health care team when making decisions at the point of care. The four major types of analytics are discussed and illustrate various applications within health care. The use of analytics promotes clinical practice that applies a data driven approach to delivering patient care services. The ability to embed analytics into clinical practice improves workflow, enhances decision making, increases productivity, reduces overall costs, and fosters optimal patient outcomes.

\section{Key words}

Nursing analytics, Predictive analytics, Health care outcomes, Quality improvement, Interprofessional education and practice

\section{I ntroduction}

There is a growing body of literature focused on the use of analytics and big data to tackle major challenges and solve complex problems in business and industry. The health care industry today-like nearly every other industry- has had to become focused on doing the most with their resources. The budgets of health care organizations have been trimmed, employees have been asked to take on more, and no longer can organizations waste resources. According to Sultz and Young $^{[1]}$ "studies indicate that $30 \%-40 \%$ of U.S. health spending is 'waste' in that it provides services of no discernible value and inefficiently produces valuable services”.

The health care professional who has knowledge of how to use analytics in clinical practice is integral to reducing costs and improving the quality of patient outcomes. The primary purpose of this article is to provide a framework that can be used for making clinical decisions using analytical data. An overview of the four major types of analytics and examples from the literature are provided to illustrate the use of analytics across various health care settings. 


\section{Overview and significance of analytics}

According to Davenport and Harris ${ }^{[2]}$ analytics involves "the extensive use of data, statistical and quantitative analysis, explanatory and predictive models, and fact-based management to drive decisions". Analytics have been used in the business sector and across industries, such as telecommunications, retail, pharmaceuticals, and consumer products over the past two decades. Analytics are often referred to as a subset of what is commonly known as business intelligence, which are processes that use data to understand and analyze business performance. Unlike standard reports and alerts, analytics uses tools to forecast, predict, and analyze patterns or trends to prevent problems and promote early action.

According to Mayer-Schoenberger and Cukier ${ }^{[3]}$ analytics are also referred to as big data, which focuses on "an understanding of the relationships within and among the various pieces of information. Big data has large scale implications and is often used to extract new insights or create new forms of values from existing data, in ways that change markets, organizations, and the relationship between citizens and governments”. Big data analytics helps an organization to differentiate itself from the competition, build capacity in the marketplace, gain a competitive advantage, and balance risk with the price of goods and services. Analytical data supports a health care system by promoting real time point of care clinical practice. The ability to embed analytics into daily processes and workflow accelerates decision making, increases productivity, reduces overall costs, and promotes a culture of quality improvement ${ }^{[4]}$.

The transformation from a service-and volume-based to a performance-and value-based health care industry is underway. Organizations that do not successfully make this transition will receive financial penalties and a reduction in reimbursement for services. For example, the Centers for Medicare Services initiated value-based purchasing in 2003 with the intent of providing "incentives to discourage inappropriate, unnecessary, and costly care" ${ }^{11]}$. Furthermore, due to requirements set forth by the Patient Protection and Affordable Care Act ${ }^{[5]}$, the "value-based purchasing program has expanded to over 3000 acute care Medicare participating hospitals, enabling them to earn incentive payments based on clinical outcomes and patient satisfaction”. These changes require informed data-driven decisions that result in improved outcomes, new insights, and innovations in clinical practice.

Analytics provides the interprofessional health care team with the capacity to "track and measure real time results in patient diagnoses and outcomes and integrate qualitative and quantitative patient reports that provide a multi-view approach to managing, treating, and caring for patient populations" ${ }^{[6]}$. For example, advanced analytical tools may "detect the diabetic patients with the highest probability of hospitalization in the following year based on age, coexisting chronic illness, medication adherence, and past patterns of care” ${ }^{[7]}$.

The volume and complexity of health information data is growing exponentially. Large and diverse forms of clinical, financial, operational, behavioral, and social data require the ability to develop new competencies in decision support ${ }^{[8]}$. The ever expanding digital universe that lies ahead provides us with opportunities to apply analytics with the goal of improving health care outcomes and drive widespread systematic changes in clinical practice. Integrating analytics into clinical practice is where the value lies. The ability to view the results of patient scenarios, manipulate analytical models, and "make decisions based on analytical insights" at the point of care changes the way in which we view clinical practice ${ }^{[8]}$.

The advancement of analytics as a way to improve patient outcomes, promote health and safety, and foster data driven decision making positively impacts the way we practice. For example, Figure 1 illustrates how analytical tools and capabilities can be used to inform members of the interprofessional health care team. Access to data, evidence, professional standards, analytical scenarios, and predictive models contribute to clinical insights and innovations in practice. In addition, an educator can readily develop standardized patient and simulation scenarios that incorporate the use of analytical data that assists with improving clinical decision making. 


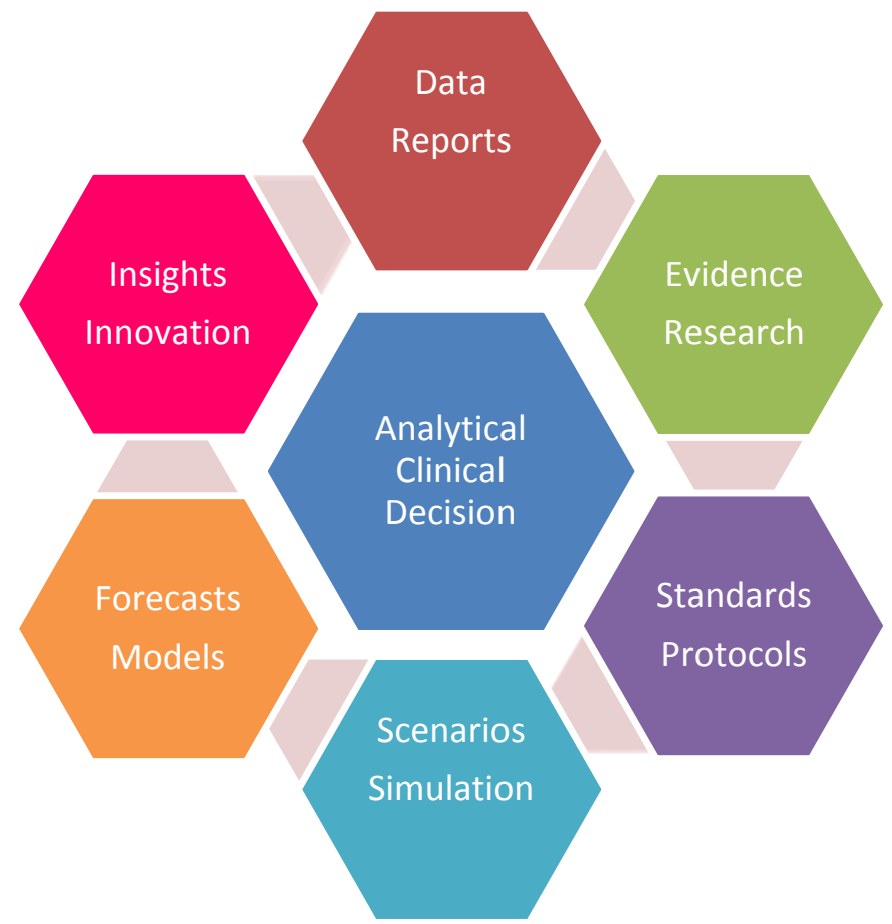

Figure 1. Analytical clinical decision framework

Analytics provide the interprofessional team with the tools needed to assess what is working and what is not, manage risk, and examine health care decisions. Thus, it promotes the coordination of patient care, reduces excessive avoidable costs, and provides accurate and reliable information at the point of care where decisions are made.

\section{Classifications of analytics}

Analytics are "classified as qualitative or quantitative according to the process employed and the type of data that are collected and analyzed. Qualitative analytics aims to gather a deep understanding of the underlying reasons and motivations for a phenomenon. Whereas, quantitative analytics uses systematic and empirical investigation of phenomena via statistical, mathematical, or computational techniques” which includes forecasting and text mining ${ }^{[9]}$.

Currently, a great deal of data in health care are unstructured and in the form of text. Therefore, it is often of limited use because it is stored internal to the organization, in formats such as e-mails, documents, patient satisfaction surveys, policies and procedures, patient management protocols, and checklists. Examples of external sources of information may consist of blogs, tweets, posts, and images. The ability to analyze and extract text, and transform it into structured, meaningful, and trusted clinical data poses significant challenges for health care organizations. However, translating unstructured text data into usable analytical data has the potential to improve every patient interaction and business process over the next decade.

Big data includes "web and social media, machine-to-machine, big transaction data, biometrics, and human generated. In addition, big data can be further delineated by volume, velocity, variety, and veracity" which all require advanced technical capabilities ${ }^{[10]}$. For example, emerging health care applications are moving from storage in terabytes to petabytes; building bigger pipes to handle the throughput of data; creating clinical, financial, and business processes that deliver data-in-motion; and transforming data from a variety of sources that are credible and suitable for clinical practice.

To date, health care organizations have not used consumer analytics as broadly as other business and industries. Reasons for this include the "lack of commercial analytical competencies, transparency, privacy concerns, and a clear mandate to do so" in the health care industry ${ }^{[8]}$. In particular, members of a health care team may have ongoing concerns related to 
what kind of information is being collected from various clinical systems. Also, concerns may be related to the accessibility of data and how it is being analyzed and shared among members of the health care team. These concerns coupled with the "need for a balance between the need for privacy [and confidentiality] with the need to have enough information to make meaningful” decisions has resulted in implementation issues ${ }^{[11]}$.

The increase in available data and information coupled with accessible portable devices are contributing to a more sophisticated consumer of health care services. Health care will continue to be shaped by the needs, wants, and preferences of consumers. The health care consumer is more analytic, comfortable with statistical data, and increasingly connected through social media. Therefore, services will be selected by consumers based on the value it provides to meet their personal health care needs now and into the future.

\section{Types of analytics}

There are four major types of analytics (descriptive, predictive, prescriptive, and comparative) that can be used to advance the development of organizational capacity and enhance decisions made by members of the health care team. Descriptive analytics use data mining to examine and analyze past events with the goal of revealing what did and did not work in the past. Predictive analytics identifies trends and patterns that can be transformed into meaningful action focused on prevention and improved patient outcomes. Prescriptive analytics goes beyond when an event will happen to why it will happen. Rather, prescriptive analytics suggests multiple courses of actions and their corresponding implications for patient outcomes. Comparative analytics are used to compare actual with expected performance outcomes through the use of metrics and national benchmarks. Knowledge of the major types of analytics equips the user with the tools necessary to analyze volumes of data, gain information and meaningful insights, and provide care focused on achieving optimal health care outcomes. Figure 2 provides a brief overview of the major types of analytics and how they can be applied to clinical practice.

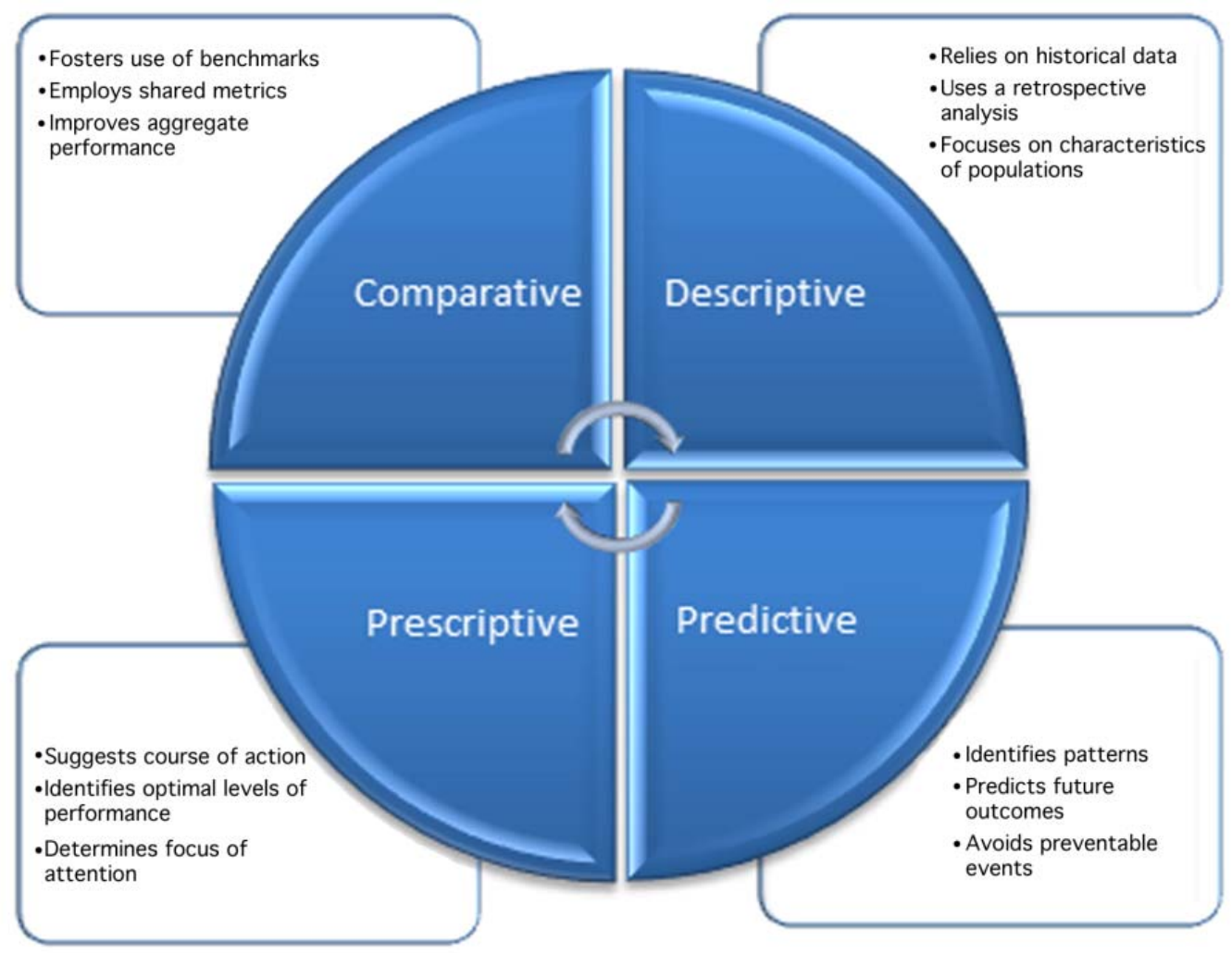

Figure 2. Four major types of analytics 


\subsection{Descriptive analytics}

Descriptive analytics involves collecting, tabulating, and mining historical data with the intent of describing the major characteristics of the population being studied. Typically, large amounts of data are examined through the lens of what went wrong, whether or not the expected outcomes were met, and how to approach the future. This accounts for the biggest amount of data across all industries and tends to be more retrospective in nature.

For example, the use of descriptive analytics would be appropriate to examine historical usage data of block-scheduling for surgical procedures in hospital operating rooms. Data on types, frequency, and length of procedures; physician availability and preferences; shift lengths; and functionality of rooms would provide valuable information on overall utilization rates. Romanyuk and Silva ${ }^{[12]}$ conducted a study using descriptive analytics to evaluate the efficiency of an operating room schedule. Initial analysis of descriptive historical data indicated that the operating rooms were used only $64.6 \%$ of the total available time. As a result, a new dynamic scheduling model emerged with the intent to improve overall utilization of the operating rooms, reduce patient wait time, and reduce hospital and patient costs.

\subsection{Predictive analytics}

Predictive analytics digs deeper into the data to identify patterns and transform them into meaningful information. This type of analytics uses technology such as modeling that "learns from experience (data) to predict the future behavior of individuals in order to drive better decisions" ${ }^{[13]}$. Predictive analytics determine the likelihood of occurrences and probable outcomes in an effort to prevent adverse events in the future. As a result, the practitioner is equipped with the tools to make timely evidence-based decisions; streamline operations and processes; and minimize risk to patients ${ }^{[14]}$.

Predictive analytics are being implemented as a result of the Affordable Care Act ${ }^{[5]}$ which went into effect on October 1 , 2012. Section 3025 of the Affordable Care Act added section 1886(q) to the Social Security Act which established the Hospital Readmissions Reduction Program. This program requires the Centers for Medicare and Medicaid Services to reduce payments to hospitals that contract with Medicare to provide acute hospital inpatient care and agree to accept predetermined acute Inpatient Prospective Payment System (IPPS) rates in full. As a result of the "Hospital Readmissions Reduction Program ${ }^{[15]}$, more than 2000 hospitals have demonstrated above-average 30-day post-discharge patient readmission rates and were penalized up to one percent of their Medicare reimbursement in 2012, resulting in a total penalty of $\$ 280$ million” ${ }^{[14]}$. The implementation of this new program in concert with the Patient Protection and Affordable Care Act is changing the current pattern of hospital readmission.

According to the U.S. Department of Health and Human Services ${ }^{[5]}$ "the all-cause 30-day hospital readmission rate among Medicare fee-for-service beneficiaries plummeted from 19 to 19.5 percent in 2011 to 17.5 percent in 2013, which resulted in an estimated 150.000 fewer hospital readmissions between January 2012 and December 2013”. Predictive analytics anticipate the likelihood of hospital readmission, mitigates risk, and thus, helps health care organizations avoid or reduce future penalties. The use of predictive analytics during the discharge planning process provide "clinical, demographic, and socioeconomic data, as well as large-scale databases which generates predictive models that identify patients that are at the highest risk for readmission" ${ }^{[3]}$. Predictive analytics reveal "correlations among patient accounts, charges, and clinical data elements that determine the attributes that result in the likelihood of readmission versus attributes that will not likely lead to readmission" ${ }^{[16]}$. With this data in hand, immediate action can be taken while the patient is in the hospital. Thus, post-discharge plans can be modified to prevent a hospital readmission and ensure successful transitions of care.

Today, the emphasis is on working in collaboration with patients and members of the interprofessional health care team to better manage chronic conditions, predict potential problems, and prevent the occurrence of disease. The reasons for this shift are substantiated by examples such as, according to the Centers for Disease Control and Prevention ${ }^{[17]}$ chronic diseases are the leading cause of death and disability in the U.S. In 2010, the Robert Wood Johnson Foundation ${ }^{[18]}$ reported that $84 \%$ of all health care spending in 2006 was for the $50 \%$ of the population who have chronic health 
conditions. Forty-five percent of Americans (133 million) have at least one chronic condition and they are responsible for seven out of 10 deaths in the U.S. killing more than 1.7 million Americans each year ${ }^{[17,18]}$.

Interrelated health conditions such as heart disease, diabetes, and obesity require highly coordinated and integrated treatment. The ability to treat and care for patients with such complex health care conditions necessitates the ability to use data from multiple sources. Predictive analytical models and metrics facilitate the gathering of information about trends, patterns, deviations, and interrelationships that foster clinical insight and innovation into the prevention and management of chronic health conditions across patient populations.

\subsection{Prescriptive analytics}

Prescriptive analytics provides "decision makers with sophisticated alternatives (insights created with speed, scale, currency, breadth, and depth) to be able to influence optimal future outcomes" ${ }^{[19]}$. The explosion of structured and unstructured clinical data has led to the need for advanced techniques that take full advantage of the vast amounts of data that facilitate planning for the future. Health care organizations are gradually transforming their core decision processes through the application of prescriptive analytics across a broad range of clinical, financial, and business functions. They automatically capture and synthesize data through warehousing, repositories, and data marts.

Prescriptive analytics uses sophisticated mathematical and statistical tools that continuously synthesize both structured and unstructured data. The value of prescriptive analytics is that it offers real time decision making models designed to improve the effectiveness of health care services with no additional data analysis by the user. For example, prescriptive analytics provides the clinician with the ability to focus like a laser beam on why the outcome will happen and how it is tied to each decision. Linking data and performance measures with core competencies such as Quality and Safety Education for Nurses (QSEN) leads to better decisions and innovation in nursing education and practice ${ }^{[20]}$. Thus, decisions occur in the context of a full range of potential health outcomes, future risks to the patient, and the long-term implications of each decision option.

The National Health Care Disparities Report ${ }^{[21]}$ tracks health care quality and disparities across the nation. This report calls for our nation to "improve access to care, reduce disparities, and accelerate the pace of quality improvement, especially in the areas of preventive care and chronic disease management. Data on often overlooked small population subgroups need to be gathered, and the burden of measurement needs to be minimized. Information needs to be disseminated more quickly to partners who have the skills and commitment to change health care". In addition, the Partnership for Patients launched in April 2011 as well as the development of national indicator datasets among several public and private organizations addresses the incidence of hospital-acquired conditions (HAC). For example, preliminary large-scale data "compiled by the Agency for Healthcare Research and Quality indicates that the HAC rate declined by nine percent from the 2010 level. This corresponds to a reduction in the measured harm rate from 145 harms per 1000 discharges in 2010 to 132 harms per 1000 discharges in 2012" "21]. Thus, the continued use of sophisticated health care data and metrics analyzed in a prescriptive manner provides valuable solutions that address health care disparities across the U.S.

\subsection{Comparative analytics}

Comparative analytics uses business intelligence to compare data among peers based on established metrics and benchmarks. Timely data provides accurate comparisons of actual patient outcomes against expected standards of care or outcomes. Thus, decisions can be made to correct or modify a treatment plan or course of action. The capability to use real time benchmark data against peer indicators for clinical and administrative performance contributes to improved patient outcomes as well as eliminates unnecessary services. As with most health conditions, it is difficult to measure and consistently compare results across hospitals and health care systems. For example, determinants such as age, severity of illness, comorbid conditions, and treatment preferences are key factors but typically are outside of the influence of the 
health care provider. However, factors that are modifiable include the relationship between providers that coexist within a select population or community and whether or not treatments for health conditions are readily available ${ }^{[22]}$.

The Joint Commission ${ }^{[23]}$ has been leading the way in the area of performance management for decades. The data collected by the Joint Commission is fostering the use of comparative analytics. For example, data that is made available on the Centers for Medicare \& Medicaid Services' Hospital Compare website reflects measures that they have in common. "A significant percentage of that data comes from The Joint Commission via its well-established performance measure data network which is comprised of approximately 39 measurement systems" ${ }^{[23]}$. The various uses of comparative analytics to improve patient care outcomes are evident in Improving America's Hospitals: The Joint Commission's Annual Report on Quality and Safety ${ }^{[23]}$ which "presents the overall performance of Joint Commission accredited hospitals on quality of care core measures relating to heart attack, heart failure, pneumonia, surgery, children's asthma, inpatient psychiatric services, venous thromboembolism (VTE), stroke, perinatal care, and immunizations”. These key measures were selected because "they provide concrete data about the best kinds of treatments or practices for common conditions for which Americans enter the hospital and seek care" ${ }^{[23]}$. The results indicate that hospitals are making steady improvement from year to year. In addition, the composite results clearly identify potential opportunities for improvement and substantiate the value of comparative measurement systems.

A consensus statement published in 2014 for Healthcare Professionals from the American Heart Association and American Stroke Association ${ }^{[22]}$ entitled "Risk Adjustment of Ischemic Stroke Outcomes for Comparing Hospital Performance" which evaluated hospital performance in providing ischemic stroke care and risk-adjusted comparisons to assess the quality of stroke care across different hospitals illustrates the value and long term potential of comparative analytics ${ }^{[22]}$. The research conducted to develop the consensus statement concluded that premorbid functioning and stroke severity are not collected in a uniform fashion in patients with stroke. Furthermore, risk-adjustment models of functional status after ischemic stroke should be standardized across health care providers so that they are reliable and accurate ${ }^{\text {[22] }}$. This consensus statement provides an opportunity to incorporate the use of comparative analytical data in curricula and professional continuing education in order to improve the identification of patients at risk for an ischemic stroke based on premorbid functional status.

\section{Conclusion}

The health care system will continue to require accessible analytical data to drive informed clinical, financial, and business decisions. Analytical decisions are the "intersection of individual and organizational capabilities" ${ }^{[24]}$. Analytics in clinical practice has the long-term potential to improve patient outcomes, promote health and safety, mitigate risk, reduce costs, address health care disparities, and promote disease prevention. Clinical decisions that are based on analytical data will benefit the individuals, families, and communities that are served by the interprofessional health care team.

\section{References}

[1] Sultz, H., \& Young, K. Health care USA: Understanding its organization and delivery. Boston, MA: Jones and Bartlett Learning. 2014. PMid:24652060

[2] Davenport, T.H., \& Harris, J.G. Competing on analytics: The new science of winning, Boston, MA: Harvard Business School Publishing Corporation. 2007. http://dx.doi.org/10.1016/j.mnl.2013.02.001

[3] Mayer-Schonberger, V., \& Cukier, K. Big data: A revolution that will transform how we live, work, and think. New York, NY: Houghton Mifflin Harcourt. 2013.

[4] Ritt, E. Embedding a culture of analytics in nursing practice. Nurse Leader. 2013; 11: 48-50.

[5] U.S. Department of Health and Human Services. Patient Protection and Affordable Care Act. 2010.

[6] CareCloud Corporation. Actionable analytics: 10 steps to improve profitability \& patient experience through data-driven decision making, Practice Performance Series White Paper, Miami, FL: CareCloud Corporation. 2012. 
[7] Wharam, J.F., \& Weiner, J.P. The promise and peril of healthcare forecasting. The American Journal of Managed Care. 2012; 18(3): e82-85. PMid:22435964

[8] Burke, J. Health analytics: gaining the insight to transform health care. Hoboken, NJ: John Wiley \& Sons. 2013.

[9] Davenport, T.H., \& Kim, J. Keeping up with the quants: your guide to understanding + using analytics. Boston, MA: Harvard Business School Publishing Corporation. 2013.

[10] Sathi, A. Big data analytics: Disruptive technologies for changing the game. Boise, ID: MC Press Online, LLC. 2013.

[11] HealthCare Analytics. Top five healthcare improvement analytics myths. 2011.

[12] Romanyuk, A., \& Silva, A. Optimization of an operating room surgical schedule.2012.

[13] Siegel E. Predictive analytics: The power to predict who will click, buy, lie, or die, Hoboken, NJ: John Wiley \& Sons Inc. 2013.

[14] Edelstein P. Emerging directions in analytics. Health Management Technology. 2013. PMid:23420986

[15] Centers for Medicare and Medicaid Services. Hospital readmissions reduction program. 2012.

[16] Bradley, P. Predictive analytics can support the ACO model. Healthcare Financial Management. 2012. PMid:22523895

[17] Centers for Disease Control and Prevention. Chronic Diseases and Health Promotion. 2014.

[18] Robert Wood Johnson Foundation. Chronic care: Making the case for ongoing care. Princeton, NJ: Robert Wood Johnson Foundation. 2012. Available from: http://www.rwjf.org/content/dam/farm/reports/reports/2010/rwjf54583

[19] Cortada, J.W., Gordon, D., \& Lenihan, B. The value of analytics in healthcare: From insights to outcomes. IBM Global Business Services Executive Report, Annual Institute for Business Value. 2012.

[20] Cronewett, L., Sherwood, G., Barnsteiner, J., Disch, J., Johnson, J., Mitchell, P., et al. Quality and safety education for nurses. Nursing Outlook. 2007; 55: 122-131. PMid:17524799 http://dx.doi.org/10.1016/j.outlook.2007.02.006

[21] U.S. Department of Health and Human Services, Agency for Healthcare Research and Quality. National health care disparities report. 2013. Available from: http://www.ahrq.gov/qual/measurix.htm

[22] Katzan, I.L., Spertus, J., Bettger, J.P., Bravata, D.M., Reeves, M.J., Smith, E.E., et al. American Heart Association Stroke Council; Council on Quality of Care and Outcomes Research; Council on Cardiovascular and Stroke Nursing; Council on Cardiovascular Radiology and Intervention; Council on Cardiovascular Surgery and Anesthesia; Council on Clinical Cardiology. Risk adjustment of ischemic stroke outcomes for comparing hospital performance: a statement for healthcare professionals from the American Heart Association/American Stroke Association. Stroke. 2014; 45 (3): 918-44. PMid:24457296 http://dx.doi.org/10.1161/01.str.0000441948.35804.77

[23] The Joint Commission. Improving America’s hospitals: The Joint Commission's annual report on quality and safety. Oakbrook Terrace, IL: The Joint Commission. 2013.

[24] Davenport, T.H., Harris J.G., \& Morison R.H. Analytics at work: Smarter decision, better results. Boston, MA: Harvard Business School Publishing Corporation. 2010. 